\title{
Urban flood modelling and planning system (UFMPS)
}

\author{
$\underline{\text { Wenhua Xu }}^{\text {a }}$, Bill Syme ${ }^{\mathrm{b}}$, Chris Huxley ${ }^{\mathrm{b}}$ and Wenyan $\mathrm{Wu}^{\mathrm{c}}{ }^{(1)}$ \\ ${ }^{a}$ Engineers Plus, ${ }^{b} B M T,{ }^{\mathrm{c}}$ The University of Melbourne \\ Email: wenhua@engineersplus.com.au
}

\begin{abstract}
There is a great need for tools to assist with complexities associated with input and output data unique to urban flood modelling. For example, it is common for stormwater asset data to be of varying levels of quality. Datasets often include many connectivity issues, unknown invert levels and wrong pipe sizes. Data errors such as these require a large amount of manual labour to rectify before they can be used as stormwater pipe network inputs for urban flood models. In addition to model input issues, the release of the ARR2016 guideline has introduced additional challenges by increasing the number of simulations required for urban flood modelling by up to a factor ten. As such, the adoption of ARR2016 requires additional work in model build, model quality control and model result processing. These challenges have triggered the need to develop a system to improve efficiency and accuracy for urban flood modelling and planning. In this paper, we discuss an urban flood modelling and planning system that automates and manages most of the challenging processes involved in urban flood modelling.
\end{abstract}

The system encompasses the whole life of urban flood modelling and planning, from model build to the delivery of flood maps and stormwater drainage AutoCAD drawings. The system's main functions include:

- The model builder: constructs models with an optimised model structure and naming convention. This allows the system to trace relevant model and result files quickly, and to perform model pre- and post-processing with the minimum user input required.

- The stormwater pipe network rationalisation tool: uses a database structure and algorithms to rationalise raw data to comply with the stormwater drainage design criteria specific to network connectivity, minimum pipe grades, maximum pipe grades, and pipe cover.

- The quality assurer element: inspects all $1 \mathrm{D}$ results to identify instability issues based on desired stability factors. It records any unstable pipes or pits in GIS format for ease of tracking. It also summarises large numbers of simulation log files to assist the identification of models that have been prematurely interrupted or were unstable.

- The pipe planner: sizes the pipes based on flows measured from targeted $1 \mathrm{D} / 2 \mathrm{D}$ model simulations.

- Other functions of the system include model pre- and post-processing for ARR2016 and ARR1987, a $12 \mathrm{~d}$ drainage model builder to check interference between the stormwater network and other underground services, an AutoCAD drawings builder to automate the output of 1D hydraulic modelling results in design drawing format and a high resolution flood animation generator linked to Google Earth.

The system is able to build a 1D and 2D coupled TUFLOW flood model, 1D Mike Urban and 12d drainage models with a rationalised stormwater pipe network within 30 minutes for an urban catchment of 1 square kilometre. Without the system, it may take 2 to 3 weeks to rationalise the pipe network for the same dataset. The system can also convert 1D stormwater networks between different flood and drainage models, to perform hydraulic modelling cross checks between different models. The system will be extended to support other models like ANUGA in the future.

The system promotes a framework for consistent model structure and naming convention. As an outcome of this work it is recommended that nationwide protocols should be made for flood modelling and stormwater asset data management. This will make flood and drainage modelling consistent nationally and potentially significantly improve modelling productivity and quality within the broader industry.

Keywords: Urban flood modelling, stormwater pipe network, ARR2016, efficiency, quality 


\section{INTRODUCTION}

Fluvial flood modelling has been widely used (Teng et al. 2017) to assess flooding of areas affected by rivers or large water bodies by ignoring stormwater drainage system and using hydrographs from traditional hydrological models for urban areas. However, stormwater drainage systems play a significant role in urban pluvial flooding assessments. As such, for accurate results stormwater drainage system details must be included in flood models to assess and address urban flood issues. Some of the challenges associated with modelling stormwater drainage systems are outlined below.

- Discrepancies in input data. It is common that flood modellers have experienced challenges when they deal with urban catchments (Liu et al. 2015). Due to the complexity of the urban drainage system and the often poor quality of the stormwater asset database, urban flood modelling requires a large amount of manual work to pre-process the stormwater network. Stormwater asset databases are different from engineering drawings, they are typically a schematic layout, and do not have sufficient accuracy to be directly used in urban flood models. For example, some inlet pits may be missing from the stormwater assets database; pipes may not be in the right direction; or only limited pipe and pit invert levels are provided. All these errors or missing information need to be rationalised before the data become usable for hydraulic analysis models. Without a suitable tool, modellers need to manually go through the whole stormwater database to find the errors and fix them. A tool was developed by DHI for City of Gold Coast to fix critical stormwater network errors in order to support Mike Urban modelling (Cornelius et al. 2012). Some 'non-critical' errors may not stop the model from running, but could lead to inaccurate modelling results. Modellers still need to work through those 'non-critical' errors and manually grade the pipe network, which is a time-consuming exercise.

- Large amount of simulations. The release of Australian Rainfall and Runoff (ARR) 2016 has significantly increased the work involved in flood modelling. First, modellers need to prepare rainfall time series data for ten temporal patterns for each duration of each Annual Exceedance Probability (AEP) flood event. Typically, simulations for 12 event durations (from 10 minutes up to 6 hours), 5 AEPs (AEP39, AEP18, AEP10, AEP5, and AEP1) and 2 climate years (current and future) are required for urban flooding assessments. Collectively this equates to 1200 events to be simulated. After the simulations, the 1200 result datasets need to be checked and processed to derive the final result. To manually prepare 600 independent rainfall datasets, and check and process so many results requires a large volume of manual labour. It can also become a source of error if interrupted or unstable simulations are not identified correctly. To further compound matters there may be a thousand stormwater pipes and pits per square kilometre in an urban area that require checking. Due to the large number of stormwater network elements in urban flood models, it's difficult to manually check all 1D results of the stormwater drainage system to ensure model quality even for a single simulation let alone 1200 simulations.

- Contributing catchment boundaries required for drainage design. One of the advantages of coupled 1D 2D urban flood models using direct rainfall hydrology is that no sub-catchment delineation is required. Unfortunately this advantage can introduce new challenges when designing new pipe network systems. Contemporary stormwater drainage system design approaches require knowledge of the upstream catchment size to estimate flow and associated design pipe sizes. Since coupled 1D 2D urban flood models do not provide this information, manual delineation of urban contributing sub-catchments is often still carried out, at least for the purpose of the initial pipe design.

All these challenges make urban flood modelling expensive, which has subsequently limited the development of urban flood models. When urban modelling projects are commissioned, local councils usually prioritise the urban catchments and only assess those with high priority status. This paper describes an urban flood modelling and planning system with the capability to address all the challenges mentioned above. The system has been used for urban flood modelling projects in the areas of Fraser Coast Reginal Council and the City of Gold Coast. The system was developed with C\# and Python (Arcpy). Most of the GIS processing is accomplished by python and general processes are undertaken by $\mathrm{C \#}$. With more advanced GIS libraries available in C\#, the system will be fully migrated to $\mathrm{C \#}$ and independent of ArcGIS for simplicity, computational and cost efficiency.

\section{SYSTEM DESIGN}

The system encompasses the whole life of urban flood modelling and planning, from model building to flood map delivery and stormwater system planning.

\subsection{System Architecture}

The system architecture follows the flood modelling process and has been developed based on relationships between different modelling tasks. The system aims to support popular flood models on the market and 
currently fully supports TUFLOW coupled 1D 2D flood models, DHI Mike Urban and 12d (a software for surveying, civil and water engineering) drainage models. The system delivers a complete TUFLOW flood model for planning purposes and provides 1D drainage models for further stormwater drainage assessment at the detailed design stage. The system also facilitates the comparison of TUFLOW and/or Mike Urban model features against $12 \mathrm{~d}$ models to check interference between the stormwater network and other underground services. It can also be used to produce AutoCAD drawings for stormwater drainage planning. Other software like ANUGA will be supported in the future.

The system architecture is outlined in Figure 1. Each module in the system architecture is explained in the following section.

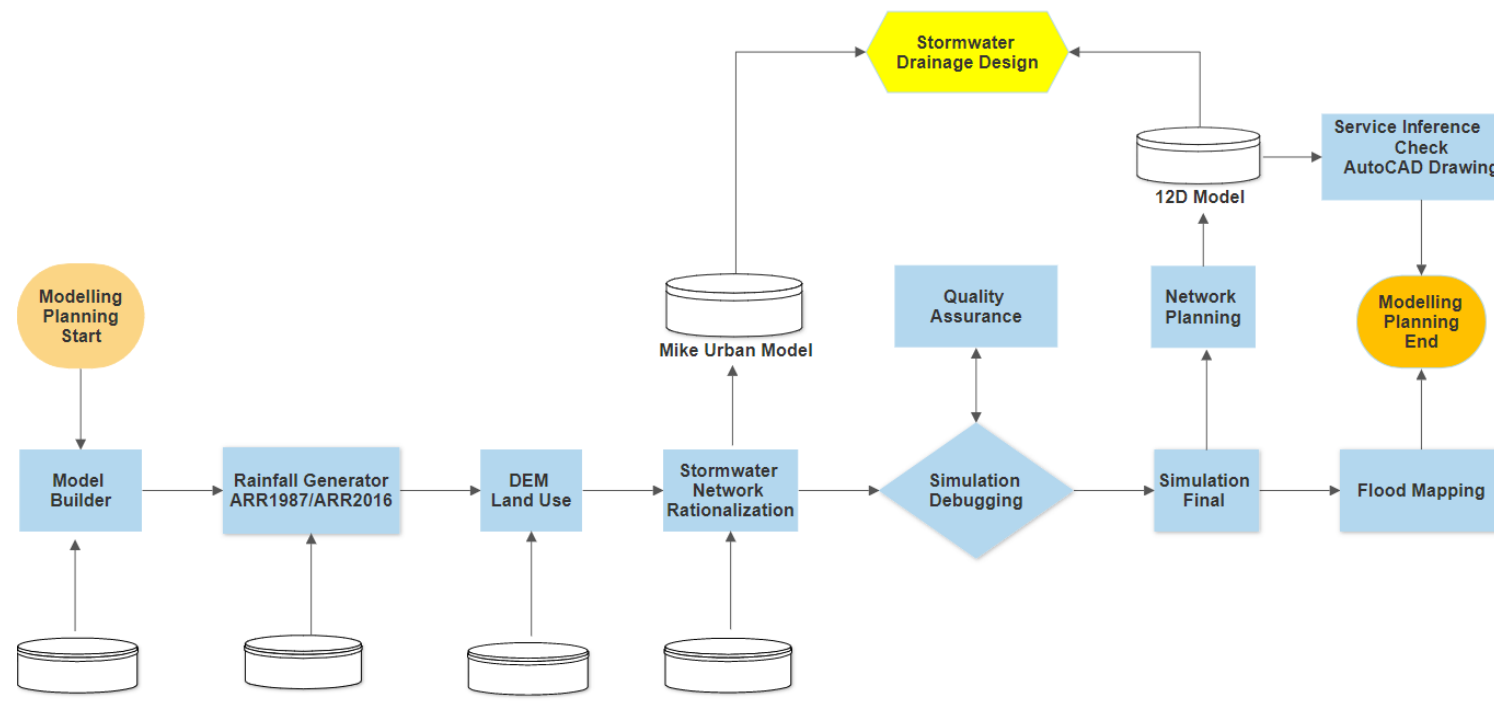

Figure 1. System architecture

\subsection{System Modules and Components}

- Model builder: builds the model framework with necessary GIS empty files and TUFLOW control files by using system data and real-time GIS templates generated by TUFLOW.

- Rainfall generator: generates rainfall for the model area from an offline ARR2016 rainfall database.

- Digital elevation Model (DEM) and land use: select a DEM file or tiles from a DEM database for the model area; prepare land use GIS from a land use database for the model area.

- Stormwater network rationalisation: checks and fixes stormwater network errors, grades the stormwater network with customised parameters, and creates 1D network inputs for TUFLOW, Mike Urban and $12 \mathrm{~d}$ models.

- Quality assurance: checks 1D results and 2D simulation log files for model stability and any errors.

- Network planning: determines critical flows from different duration simulations, sizes pipes based on critical flows, reads TUFLOW results to $12 \mathrm{~d}$ for AutoCAD drawing production and checks interference between stormwater pipes and other underground services.

- Flood mapping: determines temporal pattern resulting in the mean peak flow condition of each duration and AEP for ARR2016, produces critical duration maps for each AEP (ARR2016) or ARI (ARR1987) and analyses local temporal pattern and mean temporal pattern.

The System entry interface and all modules are shown in Figure 2.

\subsection{System Data}

The system data includes pre-supplied database for rainfall, DEM, land use and stormwater drainage network, and real-time created TUFLOW GIS template files. Local rainfall data is offline and provided by the system. Offline rainfall data is used in the system to mitigate the 'out of service' issue sometimes arising from accessing the Bureau of Meteorology's (BOM) online Intensity-Frequency-Duration (IFD) and ARR data. Also the BOM online IFD and ARR datahub have changed data format previously, which caused issues for other software sourcing the live online data. Offline data provides contingency for both such events. 


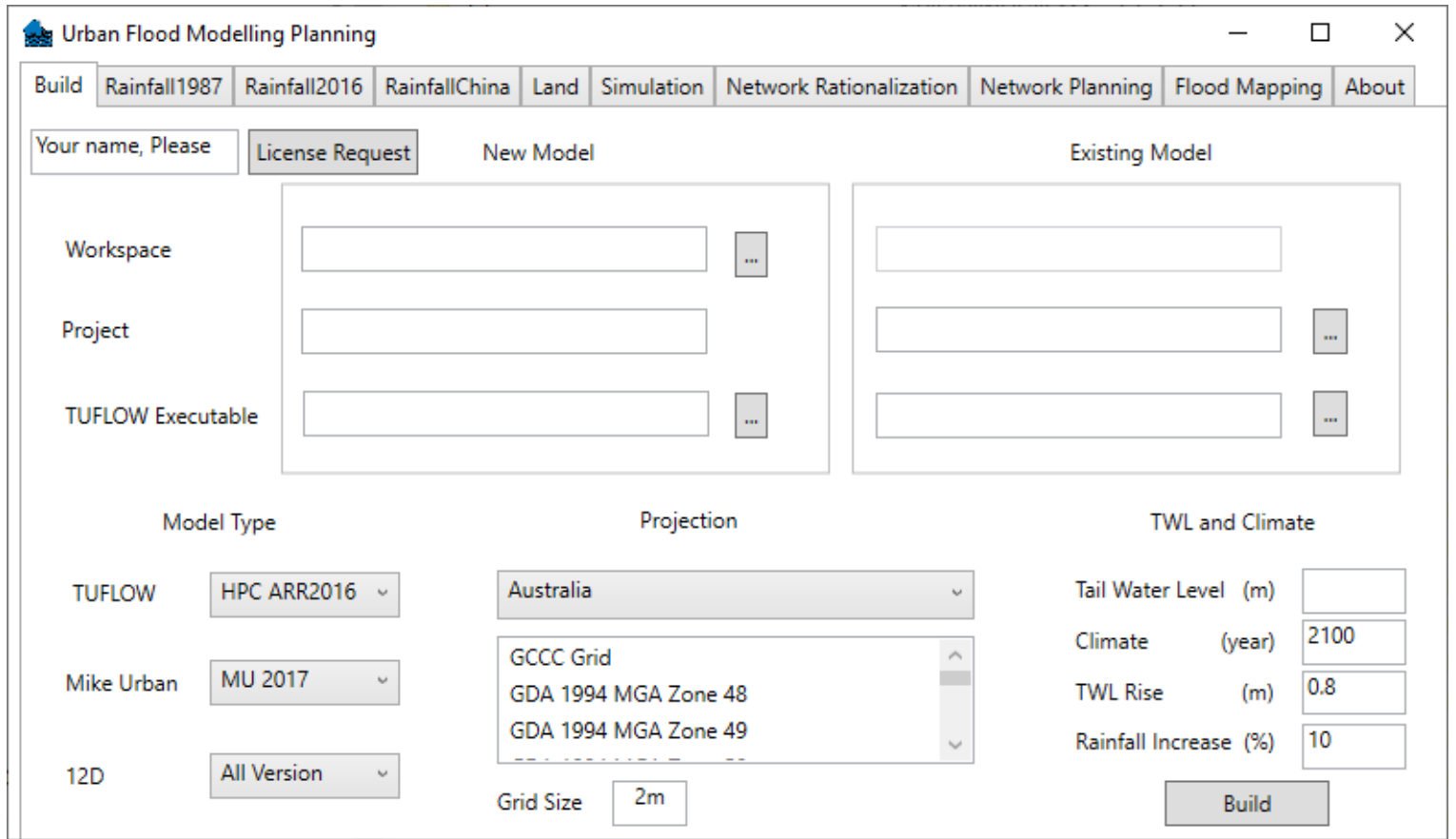

Figure 2. System interface

\section{SYSTEM IMPLEMENTATION}

The system is implemented with the maximum automation for complex model pre- and post-processing. If all databases are prepared and formatted as per system templates, the only manually entered datasets required are the 2D domain polygon and catchment boundary. All other TUFLOW 2D model GIS and control files are automatically generated from the system data and based on model parameters.

\subsection{Model Build}

Model build plays an important role in the system. It creates an integrated model environment for information exchange between modules. The flood model folder structure and file naming conventions are in compliance with project names and scenarios. This maintains easy traceability of model GIS files and result files. It also supports model pre- and post-processing to be completed with minimal user input. The local projection system for the modelling area, tail water level condition and climate change parameters are specified in this module. All model GIS template files are created by TUFLOW based on the selected projection system. All TUFLOW control files are customised from these template files. A 2D domain polygon (2d_domian layer) and a catchment (2d_code layer) polygon are required to build the TUFLOW 2D model. Once model domain and catchment are digitised, the system is ready to automatically generate rainfall and other model GIS files. In addition to the TUFLOW model creation, model build also creates an ArcGIS workspace of all the spatial inputs. It also includes functionality for users to easily review the parameters of existing models.

\subsection{Rainfall ARR 2016, Rainfall ARR 1987 and Rainfall China}

This module generates design rainfall time series data for a TUFLOW model for specified AEPs and durations.

As mentioned in the Section 2.4, the ARR2016 rainfall and temporal pattern database is available offline for data reliability. ARR2016 rainfall data for 10 temporal patterns of each duration and AEP are generated. The module also separately extracts the closest temporal pattern to the catchment (the local temporal pattern). The local temporal pattern is primarily used for model debugging prior to the mean temporal pattern being identified. The local and mean temporal patterns are further discussed in Section 3.7. Rainfall GIS files are generated with factors reflecting different climate scenarios. A single rainfall polygon with average rainfall intensity can be used for small catchments and rainfall grids representing spatial variation in rainfall intensity is suitable for large catchments. Both rainfall options are included in the TUFLOW model boundary control file and can be chosen depending on the catchment size.

Rainfall 1987 is simpler than ARR2016. Before BOM retires ARR87 IFD, IFD data can be extracted from the BOM website. The system generates ARR1987 rainfall time series data for a TUFLOW model based on that IFD and selected temporal pattern. 
The system is also capable of generating rainfall data based on project locations in China. IFDs can be generated based on formulas for different cities of China, and temporal patterns can be derived from the Chicago temporal pattern approach or customized local temporal pattern.

\subsection{Land}

This module prepares the input GIS data for the flood model, which include elevation, land use and downstream (tail water) boundaries.

Elevation data are defined using a DEM. This could be a single DEM file or multiple DEM tiles. For DEM tiles, a shape file is prepared for tile indexing. The tiles within the catchment or intersecting with the catchment boundary are selected and copied to the model folder, and the TUFLOW geometry control file is automatically updated with reference to the selected DEM tiles.

The material GIS layer for flood models are generated for the modelling area from the land use database. The material code and land use name are automatically mapped. The TUFLOW material file generated by the system automatically matches the material GIS layer.

The 2D tail water boundary GIS layer is also generated in this module by converting the catchment polygon to a polyline and assigning the boundary name from tail water level database generated in 'Model build'. The default 2D tail water boundary GIS generated by the system may be modified or overwritten if the automatically generated option is not desirable.

\subsection{Simulation}

The completion of tasks through module one to module three will produce a TUFLOW 2D model which can be simulated for selected rainfall events and climate scenarios. The local temporal pattern is used to simulate and debug the model. A batch file for simulations of all rainfall events specified in the Rainfall module is also created.

Two functions are included for quality assurance:

- Stability check of 1D results: Peak values of flow and velocity during TUFLOW simulations are recorded in $1 \mathrm{D}$ result files together with time series data. By comparing a desired stability factor with the ratio between the peak value and the maximum value in the time series data, we can identify any instabilities within the $1 \mathrm{D}$ results.

- Summary of TUFLOW simulation log files: Key words are used to summarise TUFLOW simulation log files. Any unstable or interrupted simulations are reported in a separate csv file for attention. Numbers of 1D and 2D negative depths, repeat time steps and NaN ('Not a Number') (TUFLOW, 2019) for all simulations are reported in another csv file.

These quality assurance functions provide a summary for users to review critical information about hundreds of simulations in one place.

\subsection{Network Rationalization}

Network rationalization addresses the most challenging element of urban flood modelling. It was also the most difficult part of the system to implement. The module has been designed to identify and fix stormwater network errors, grade the network, and prepare 1D pipe network for TUFLOW, Mike Urban and 12d drainage models.

Typical network errors and relevant rectification actions completed by the module are listed below:

- Missing pits are identified and recorded in GIS. They need to be manually added and categorised as manholes, inlets or outlets.

- Standalone nodes are identified and recorded in GIS. They can be automatically removed.

- $\quad$ End manholes which are located at downstream end of pipes are recorded in GIS. This typically indicates one of the connected pipes to the manhole has been digitised in the wrong direction and needs to be manually reversed.

- Outlet structures located in the middle of the network are recorded in GIS. In this situation the network needs to be manually broken at the outlet structure, and a new inlet structure added in the broken network.

- Pipes not broken at junctions are recorded in GIS. They can be automatically broken at the nearest junctions and snapped to them.

Some potential network errors are also identified and recorded with GIS files during rationalization. In some situations, errors need to be rectified. While in other situations, they may be correct. For example: 
- The network ends at an inlet pit. If the pit is a normal inlet pit, it highlights an error indicating the pipe is digitised in the wrong direction. If the pit is a surcharge pit in open space, it is correct.

- $\quad$ Pipes are too deep or too shallow.

- Downstream pipes' total cross section area is less than upstream pipes' total cross section area.

Once all network errors and potential errors are rectified, the network can be graded based on minimum pipe grade, maximum pipe grade, and minimum soil cover specifications for different pipe sizes, types and classes. The maximum pipe grade for different size pipes is integrated in pipe networks based on the QLD Urban Drainage Manual (QUDM) and cannot be changed. All other parameters are stored in a text file and can be customized. The pipe grading can be traced from upstream to downstream or in the reverse direction. By default, pipes are graded from upstream to downstream. Known invert levels from survey, design or as-construction data will be respected to different degrees by flagging the feature. They can be fixed if the levels are known to be accurate, or can be revised if they do not meet stormwater design criteria. Repeated grading may occur, though sometimes nothing may be updated. To avoid unwanted regrading, the system flags all graded pits and pipes and compares the current graded invert level with the previous graded invert level. If the current graded invert level is higher than or equal to the previous graded invert level, the system will break the grading from this point. Otherwise, the system will continue grading the next downstream invert level. This automated workflow significantly reduces

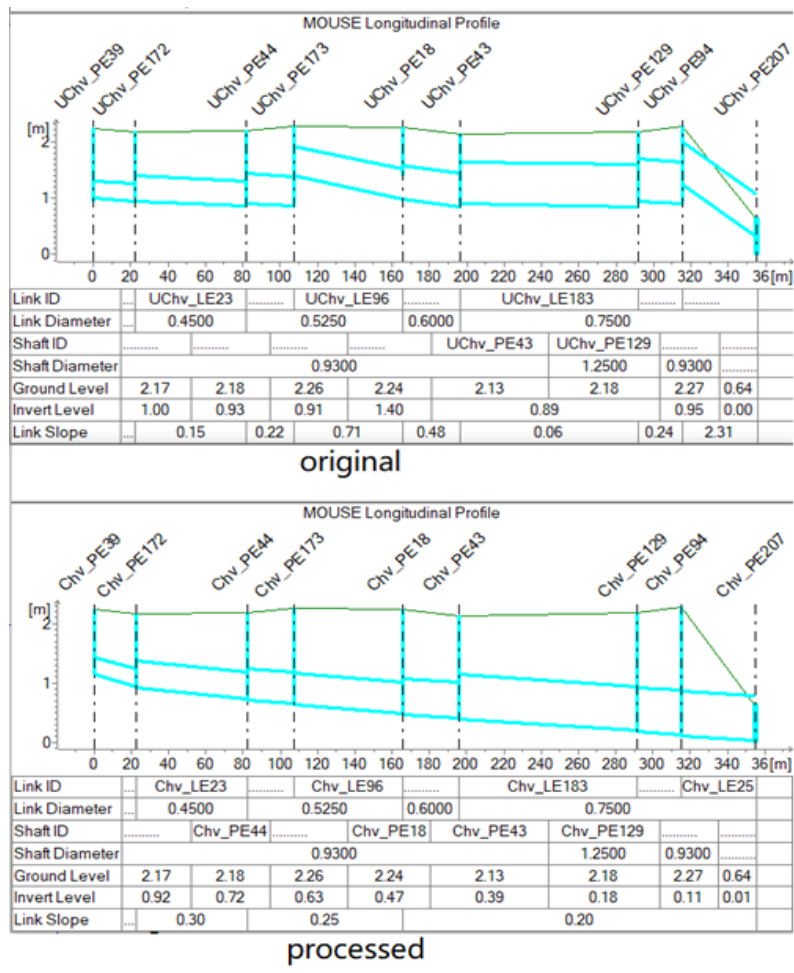

Figure 3. Pipe network grading network grading time.

The module creates 1D stormwater network inputs for TUFLOW, Mike Urban and 12d models. All network configurations are automatically defined based on information from the stormwater asset database and graded network (e.g. pipe size, kerb and field inlet size, manhole size, and invert levels). Pipe exit and entrance loss coefficients are assigned based on what the pipe is connected to. Figure 3 shows the original and processed pipe network in Mike Urban.

\subsection{Network Planning}

Network Planning aims to assist with the design of new pipe network systems to accommodate a designated flow condition. The system completes this task by initially oversizing the pipes. The objective of using oversized pipes aims to fully capture the flows in the 1D stormwater network. The storage of the oversized system is adjusted to a minimum to maintain model stability.

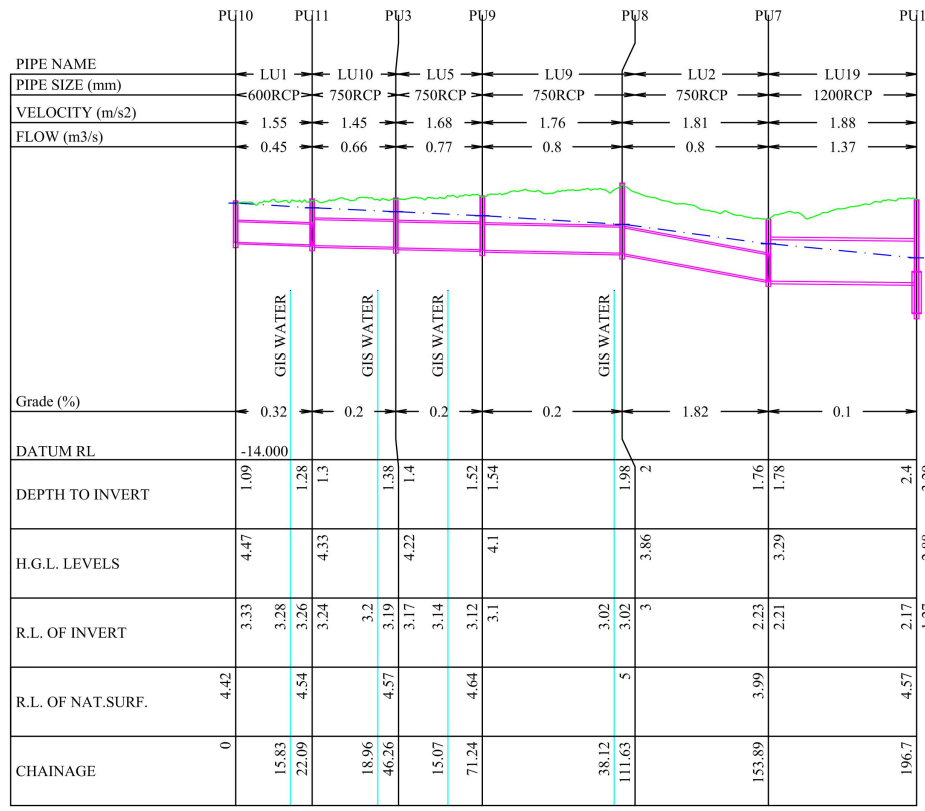

Figure 4. AutoCAD drawing

For ARR2016, the temporal pattern resulting in the mean peak flow condition for each duration is identified first. The critical duration for each design AEP is then identified and used to size the pipes. 
The system provides an efficient interface between flood modelling and engineering. A $12 \mathrm{~d}$ drainage model is built with the same stormwater network as the TUFLOW and Mike Urban models. TUFLOW modelling results are retrieved and written to the $12 \mathrm{~d}$ model. Within the $12 \mathrm{~d}$ model, the interference between the planned stormwater system and other underground services can be checked for engineering purposes. Further to that, from the $12 \mathrm{~d}$ model, standard stormwater drainage AutoCAD drawings are produced with necessary TUFLOW modelling features and results like pipe flow, pipe velocity and hydraulic grade line. An example of the standard AutoCAD drawing produced by the system is shown as Figure 4.

\subsection{Flood Mapping}

For ARR2016, mean depth, level or hazard flood maps are calculated for each duration and each AEP for the whole catchment. Then critical flood maps among different durations for each AEP are calculated. The system also determines mean temporal patterns, local temporal patterns and critical durations for sub-catchments and records the information in a csv file. The temporal pattern and critical duration information of the subcatchments can be used for next stage detailed stormwater modelling.

It should be noted that ARR2016 recommends that the temporal pattern translating to the mean peak flow result should always be used. It is the lead author's view that the peak flow of the local temporal pattern should be compared with that of the mean temporal pattern and the conservative one should be used. Therefore, this system identifies the mean temporal pattern and the conservative temporal pattern for users to choose.

The system uses GDAL to generate Google Earth overlay tiles for high resolution flood animations. This is a step forward than using normal GIS packages like ArcGIS that can only generate a single coarse resolution KML file at a time for flood animations.

\section{SYSTEM APPLICATION}

The system has been used for urban flood modelling projects in the areas of Fraser Coast Regional Council and City of Gold Coast. For coupled 1D 2D TUFLOW urban flood modelling of a medium-sized urban catchment, the delivery time would be up to six months for ARR1987 and eight months for ARR2016, if the tasks were carried out manually. With assistance of this system, we have achieved higher confidence in modelling results and reduced the delivery time of an ARR1987 flood modelling project to three weeks. An ARR2016 flood modelling project may require a two-month timeframe due to the significant increase in the number of simulations required, noting that the man-hours required for ARR1987 and ARR2016 flood modelling projects would be similar if using this system.

The system can also help local government to rationalise their stormwater drainage network for general purposes, for example if the cost for stormwater network survey is impractical.

\section{CONCLUSION}

The urban flood modelling and planning system addresses the complexities of urban flood modelling, and significantly improves the quality and efficiency in model building. It is recommended that protocols should be created and adopted for flood modelling and stormwater asset data management. This will make flood and drainage modelling consistent across jurisdictions and has the potential to significantly improve modelling quality and productivity.

\section{ACKNOWLEDGMENTS}

This system was used for the projects initiated by Kane Macready - Director of Engineers Plus, demonstrated to and shared with water professionals around the world. The author acknowledges contributions from all people helping to test the system.

\section{REFERENCES}

Teng, J., Jakeman, A.J., Vaze, J., Croke, B.F.W., Dutta, D. and Kima, S. (2017). Flood inundation modelling: A review of methods, recent advances and uncertainty analysis. Environmental Modelling and Software 90, 201-216.

Liu, L., Liu, Y., Wang, X., Yu D., Liu K., Huang, H., and Hu G. (2015) Developing an effective 2-D urban flood inundation model for city emergency management based on cellular automata. Nat. Hazards Earth Syst. Sci. 15, 381-391.

Cornelius, S., Mirfenderesk, H. and Chong, E. (2012). 2012 Floodplain Management Australia National Conference.

TUFLOW Classic/HPC User Manual, Build 2018-03-AD (2019). BMT. P10-3 and P10-4. 